\title{
Absolute charge calibration of scintillating screens for relativistic electron detection
}

\author{
A. Buck, ${ }^{1,2, a)}$ K. Zeil, ${ }^{3}$ A. Popp,,$^{1,2}$ K. Schmid, ${ }^{1,2}$ A. Jochmann, ${ }^{3}$ S. D. Kraft, ${ }^{3}$ B. Hidding, ${ }^{4}$ \\ T. Kudyakov, ${ }^{4}$ C. M. S. Sears, ${ }^{1}$ L. Veisz, ${ }^{1}$ S. Karsch,,${ }^{1,2}$ J. Pawelke,${ }^{3,5}$ R. Sauerbrey, ${ }^{3}$ \\ T. Cowan, ${ }^{3}$ F. Krausz, ${ }^{1,2}$ and U. Schramm ${ }^{3}$ \\ ${ }_{1}^{1}$ Max-Planck-Institut für Quantenoptik, Hans-Kopfermann-Straße 1, D-85748 Garching, Germany \\ ${ }^{2}$ Ludwig-Maximilians-Universität München, Am Coulombwall 1, D-85748 Garching, Germany \\ ${ }^{3}$ Forschungszentrum Dresden Rossendorf, Bautzner Landstraße 400, D-01328 Dresden, Germany \\ ${ }^{4}$ Institut für Laser und Plasmaphysik, Heinrich-Heine-Universität Düsseldorf, Universitätsstraße 1, \\ D-40225 Düsseldorf, Germany \\ ${ }^{5}$ Oncoray, Technische Universität Dresden, Fetscher Straße 74, D-01307 Dresden, Germany
}

(Received 9 November 2009; accepted 18 January 2010; published online 3 March 2010)

\begin{abstract}
We report on new charge calibrations and linearity tests with high-dynamic range for eight different scintillating screens typically used for the detection of relativistic electrons from laser-plasma based acceleration schemes. The absolute charge calibration was done with picosecond electron bunches at the ELBE linear accelerator in Dresden. The lower detection limit in our setup for the most sensitive scintillating screen (KODAK Biomax MS) was $10 \mathrm{fC} / \mathrm{mm}^{2}$. The screens showed a linear photon-to-charge dependency over several orders of magnitude. An onset of saturation effects starting around $10-100 \mathrm{pC} / \mathrm{mm}^{2}$ was found for some of the screens. Additionally, a constant light source was employed as a luminosity reference to simplify the transfer of a one-time absolute calibration to different experimental setups. (C) 2010 American Institute of Physics.
\end{abstract}

[doi:10.1063/1.3310275]

\section{INTRODUCTION}

After their theoretical prediction three decades ago, ${ }^{1}$ laser-plasma based electron accelerators in the laser wakefield acceleration (LWFA) regime have evolved and are now able to produce monoenergetic electron bunches with energies between several $\mathrm{MeV}$ and $1 \mathrm{GeV}$ and charges of 1-100 $\mathrm{pC}^{2-8}$

Beam charge and energy spectrum are the most important parameters to obtain when characterizing this new type of relativistic electron sources. Detection methods for these beam parameters that have been developed for state of the art radio frequency linear accelerators (LINACs) have been partially adopted for relativistic electrons generated by this new acceleration scheme. However, the beam parameters of laserplasma based accelerators are currently quite different from LINACs that typically produce electron bunches in the $\mathrm{MeV}$ to $\mathrm{GeV}$ range with a very narrow energy distribution and charges on the order of tens of $\mathrm{pC}$ to $\mathrm{nC}$ at repetition rates up to the megahertz range. Additionally, single-shot detection methods are crucial for laser accelerated electrons because of strong fluctuations in the electron bunch parameters in consecutive pulses.

Two established devices for energy-independent singleshot bunch charge measurements in LWFA are Faraday cups and integrating current transformers (ICTs). However, these detectors do not provide information about the energy spectrum of the accelerated bunch, which can fluctuate significantly. Also they measure not only the charge in the monoen-

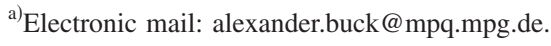

ergetic peak but also the low energy tail of the electron spectrum that can contain more charge than the peak itself. Finally, electromagnetic pulses generated in the laser-plasma interaction distort these measurements, usually limiting ICT measurements in LWFA experiments to charges of $>10 \mathrm{pC}$.

Energy-resolved charge distributions are measured with electron spectrometers (ESMs) consisting of permanent or electromagnets and a charge sensitive detector. The dipole magnet introduces an energy dependent dispersion such that the electron energy is translated to position on the detector. Unlike spectrometers for rf accelerators, which have to measure the electron energy with good resolution in a very narrow energy range, ${ }^{9}$ ESMs for laser-plasma based accelerators need to cover a wide energy range and provide an energy resolution of only about $1 \% .^{10-15}$ Thus, the detection plane at the exit of the ESM often extends over several hundred $\mathrm{cm}^{2}$.

Suitable candidates for the electron detection are scintillating materials (fibers or screens) or imaging plates (IPs). ${ }^{13,14,16}$ IPs have an active layer of tiny crystals $(\sim 5 \mu \mathrm{m})$ that can store high energy radiation. The IPs must be scanned after the experiments in order to read out the data, making them unpractical for experiments with high repetition rate. Scintillating fibers that are directly coupled to a charge-coupled device (CCD) camera can provide highdynamic range ${ }^{8,15}$ but have a limited energy resolution because of the fiber thickness and give no information about beam divergence. Therefore, scintillating screens with a layer of powdered rare earth phosphor that are imaged to a CCD camera are typically used for the detection of LWFAelectrons.

The linear response of these scintillating screen detectors 
TABLE I. Results of absolute calibration, cross-calibration to CLS, and saturation thresholds for all scintillation screens.

\begin{tabular}{lccc}
\hline \hline Screen & $\begin{array}{c}\text { Absolute calibration } \\
\left(10^{9} \text { photons/sr/pC) }\right.\end{array}$ & $\begin{array}{c}\mathrm{N}_{\text {scint }} / \mathrm{N}_{\text {CLS,20 ms }} / \mathrm{Q} \\
\left(\mathrm{pC}^{-1}\right)\end{array}$ & $\begin{array}{c}\rho_{\text {sat }}(\text { see Sec. III C) } \\
\left(\mathrm{pC} / \mathrm{mm}^{2}\right)\end{array}$ \\
\hline KODAK Biomax MS & $14.8 \pm 1.3$ & $5.79 \pm 0.26$ & $21.8 \pm 5.0$ \\
CAWO OG 16 & $12.4 \pm 1.1$ & $4.86 \pm 0.21$ & $32.9 \pm 6.6$ \\
KODAK Biomax Transcreen HE & $7.85 \pm 0.67$ & $3.02 \pm 0.13$ & $47 \pm 10$ \\
KODAK Lanex Regular & $6.95 \pm 0.60$ & $2.72 \pm 0.12$ & $66 \pm 33$ \\
KONICA KR & $6.58 \pm 0.56$ & $2.58 \pm 0.11$ & $>100$ \\
KODAK Biomax Transcreen LE & $1.79 \pm 0.15$ & $0.700 \pm 0.031$ & $>100$ \\
KODAK Lanex Fine & $1.75 \pm 0.15$ & $0.686 \pm 0.030$ & $>100$ \\
KONICA KF & $1.54 \pm 0.13$ & $0.602 \pm 0.027$ & $>100$ \\
\hline \hline
\end{tabular}

to the short LWFA-electron bunches with pulse durations below 100 fs and charges that can reach up to several $100 \mathrm{pC}$ has been heavily discussed. The ELBE linear accelerator at the Forschungszentrum Dresden-Rossendorf can deliver pulse trains of variable length at $1 \mathrm{kHz}$ repetition rate with a micropulse repetition rate of $13 \mathrm{MHz}$. The single electron bunches have energies of up to $40 \mathrm{MeV}$, tunable charges of up to $80 \mathrm{pC}$, and a pulse duration of $2 \mathrm{ps}$. The irradiation site has been optimized for highest possible peak intensity including precise bunch charge measurements with multiple cross-calibrated charge monitors ${ }^{16}$ and is therefore ideally suited to simulate the LWFA conditions.

We have performed linearity tests and absolute charge calibrations for eight different scintillating screens. The calibration results show the advantages of the different screens and can be applied to other electron acceleration setups. However, after applying the calibration, any change in the imaging geometry will result in a loss of accurate charge calibration, making it necessary to recalibrate the imaging setup. Therefore, we have also cross-calibrated the scintillating screens to a constant light source (CLS).$^{10}$ The calibration setup and the CLS are explained and characterized in Sec. II. Section III describes the results of the absolute charge calibration of the screens, cross-calibration to the CLS, and the investigation of the screen linearity. The results are discussed in Sec. IV.

\section{SETUP}

\section{A. General}

The calibration measurements of the scintillating screens (Table I) were performed at the ELBE linear accelerator, which was tuned to electron bunch charges of up to $50 \mathrm{pC}$ with an energy of $40 \mathrm{MeV}$. For charges above $50 \mathrm{pC}$, pulse trains with tunable length and $154 \mathrm{~ns}$ delay between the single pulses were used to increase the calibration charges up to $100 \mathrm{nC}$. Although the accelerator can run at megahertz repetition rate, only $0.1 \mathrm{~Hz}$ was used for the electron pulses or pulse trains during the calibration measurements. Thus, the maximum electron beam flux was below $10 \mathrm{nA} / \mathrm{cm}^{2}$, and the scintillating screens were not damaged during the measurements. The calibration setup is shown in Fig. 1(a). The electrons exit the accelerator through a beryllium window and hit the scintillating screen at $0.38 \mathrm{~m}$ distance. Simulations and measurements show that the energy deposited in
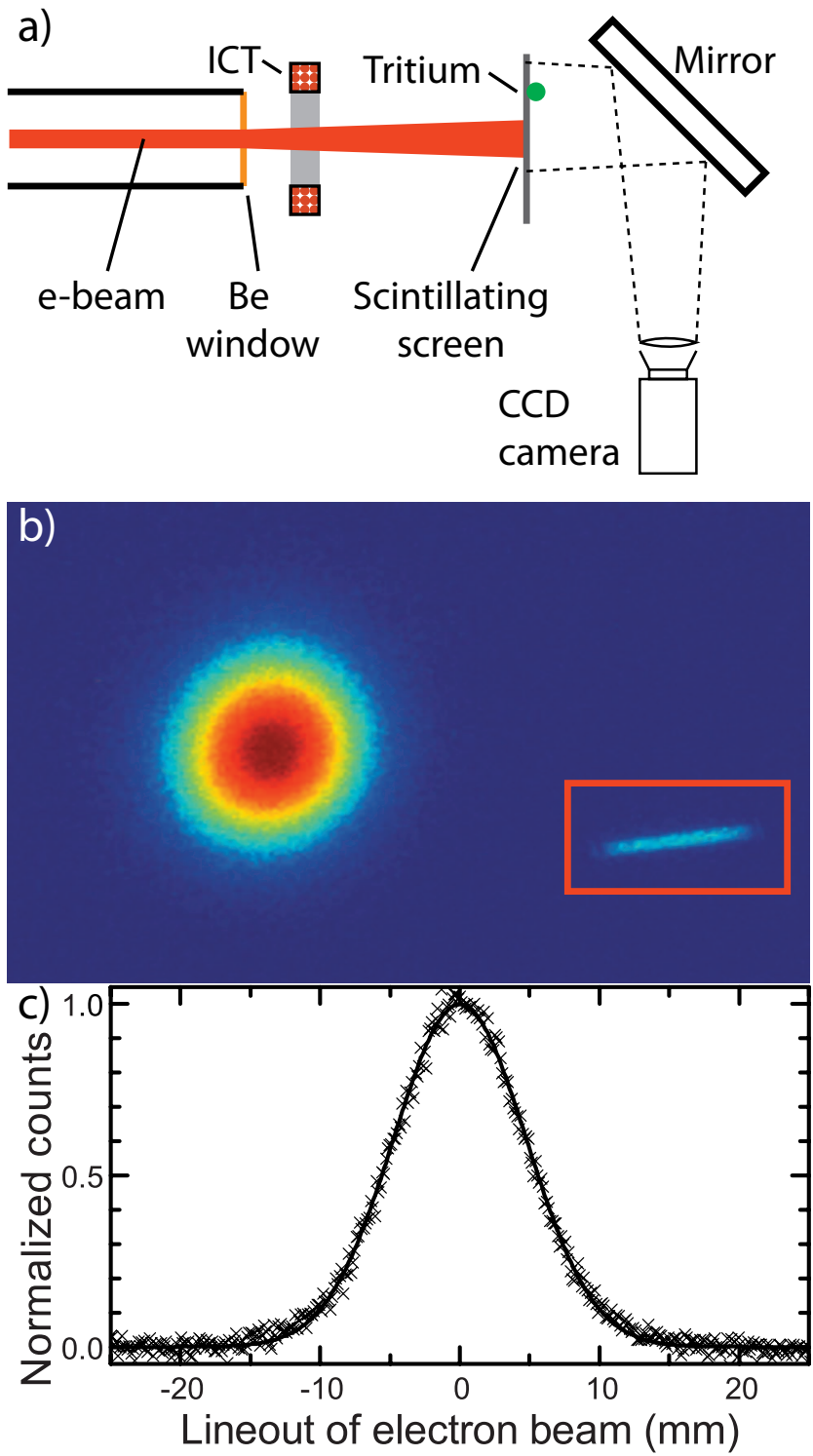

FIG. 1. (Color) (a) Calibration setup: The electrons exit the LINAC through a Beryllium window and hit the scintillating screen at $0.38 \mathrm{~m}$ distance. A CLS is placed on the scintillating screen for calibration. The scintillation signal and the CLS are imaged onto a CCD camera. The bunch charge is measured between LINAC and the screen with an ICT. (b) Typical CCD image of electron bunch and CLS. The area used for the CLS calibration is marked with a red rectangle. (c) Example for Gaussian fit of a horizontal lineout through the peak in (b). 

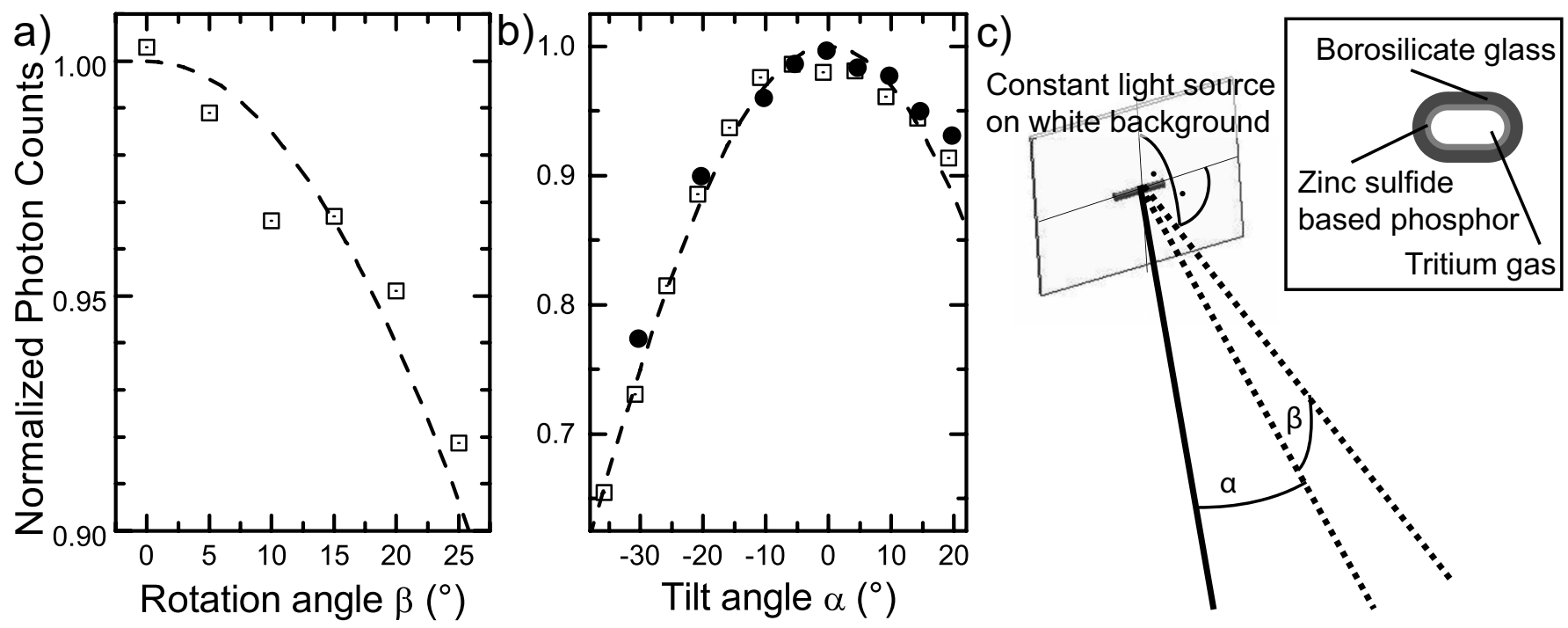

FIG. 2. Directional characteristic of the CLS with rotation around CLS axis (a) or tilting the camera toward the base areas of the CLS (b) [a white paper background (open squares) or Lanex background (filled circles)]. A Lambertian law and a cosine-squared law are fitted for (a) and (b), respectively, to guide the eye. (c) Illustration of the rotation angles for (a) and (b); $\beta$ marks a rotation around the CLS axis; $\alpha$ marks a rotation toward the side. Inset: Cross-section of the CLS

the scintillating screens per electron is almost constant for electron energies above $3 \mathrm{MeV} ;{ }^{11-13}$ thus the calibration can be applied to all relativistic electrons above this threshold produced by current laser-plasma accelerators.

The bunch charge is measured between LINAC and screen with an ICT (ICT-050-070-5:1, Bergoz Instrumentation, Saint Genis Pouilly, France). The systematic error of the ICT measurement has been estimated to be about $3 \%$ by comparing the calibration to another ICT with different readout electronics and to a Faraday cup. ${ }^{16}$

A CLS (see Sec. II B) is placed on the scintillating screen for cross-calibration. The scintillation signal and the CLS are imaged onto a CCD camera (SCOR-20SOM-CS, Point Grey, Richmond, BC, Canada) [Fig. 1(b)].

The light emission of the scintillating screens approximately follows a Lambertian (cosine) law. ${ }^{17}$ The camera looks perpendicularly at the screens. The exposure time was $20 \mathrm{~ms}$ to ensure that the scintillating screen had decayed completely and that all of the scintillation signal was recorded. Background pictures including the dark current of the accelerator were recorded and subtracted accordingly. An adjustable iris was used to increase the dynamic range of the measurement. Each screen was measured with electron bunch charges varying over four orders of magnitude. The electron signal on the CCD image is fitted well with a Gaussian curve; an example lineout is shown in Fig. 1(c). The total number of counts and the peak counts are calculated from the Gaussian fit to be less sensitive to noise in the images. The electron beam has a total divergence of $28 \mathrm{mrad}$ fullwidth at half-maximum (FWHM).

\section{B. Constant light source (CLS)}

A CLS is used for the cross-calibration measurement. For this purpose we use a cylindrical glass capsule of $12 \mathrm{~mm}$ length and $2 \mathrm{~mm}$ diameter filled with tritium and covered with scintillating material (mb-microtec) [inset of Fig. 2(c)]. The CLS is placed directly on the scintillating screen. A typical image of the CLS and the area over which the photons are counted is displayed in Fig. 1(b). The photon flux from the CLS is then measured relative to the scintillation signal from the screens for cross-calibration. The tritium in the capsule decays exponentially with a decay time 8.52 years. However, the luminosity of this tritium capsule decreases faster than the exponential decay because of the degradation of the scintillating material. The drop in luminosity is not exponential but can be estimated with an exponential decay with 5 years decay time. However, the error can be up to $10 \%$ after 1 year, making it necessary to recalibrate the CLS. ${ }^{18}$

The CLS on the white background does not radiate isotropically. The directional characteristic was measured in a separate setup [Fig. 2(c)]. When rotating around the axis of the CLS [angle $\beta$ in Fig. 2(a)], the emission was found to follow a Lambertian law (cosine). When rotating toward the axis [angle $\alpha$ in Fig. 2(b)], the measured signal drops faster. A cosine-squared function fits the directional characteristic well up to $40^{\circ}$ deviation from normal incidence. Therefore, it was taken care that the photon flux both from the CLS and the scintillating screens was always measured perpendicularly to the scintillating screens $\left(\alpha=\beta=0^{\circ}\right.$ in Fig. 2).

\section{CALIBRATION RESULTS}

\section{A. Absolute calibration}

We have absolutely calibrated our imaging system to convert the counts detected by the CCD camera back into the number of photons emitted by the scintillating screen. We have used an objective with a measured focal length of $(21.68 \pm 0.15) \mathrm{mm}$ and a diameter of the effective aperture of $(7.6 \pm 0.2) \mathrm{mm}$. The objective was placed in a distance of $(589.7 \pm 6.1) \mathrm{mm}$, which results in a solid angle of $(1.394 \pm 0.074) \times 10^{-4}$ sr. For this small angle, the directional characteristic of the scintillating screen can be neglected. 


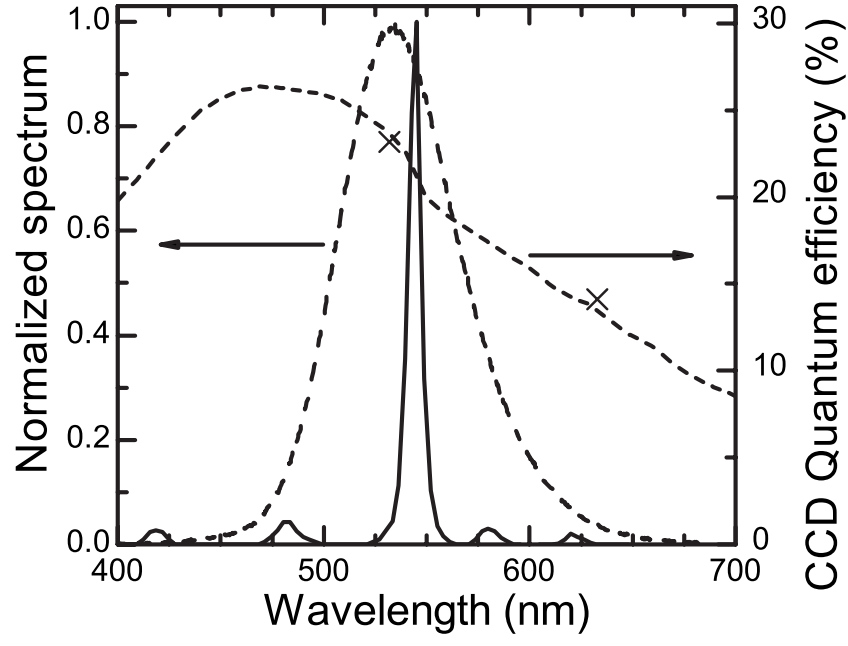

FIG. 3. Emission spectra of Kodak Lanex Regular screen (solid line) and CLS (dashed line) in comparison to the quantum efficiency of the CCD camera (dotted line). The two crosses mark measured points of the quantum efficiency.

The emission spectrum of the scintillating screens has a sharp peak at $546 \mathrm{~nm}$ (Fig. 3). At this wavelength, the transmission of the objective has been measured to be $(76 \pm 1) \%$. The quantum efficiency of the CCD (photons-to-counts) has been measured for two different wavelengths to scale the CCD efficiency curve from the manufacturer for the camera parameters used in the experiment (Fig. 4). The efficiency curve is multiplied with the emission spectrum of the scin- tillating screens to obtain the total efficiency of $(20.8 \pm 1.1) \%$. A relative error of $5 \%$ is assumed for the total efficiency because the efficiency curve does not overlap with both measured values perfectly. The reflectivity of the silver mirror is $(98 \pm 1) \%$.

The calibration curves for all the eight different screens are shown in Fig. 4. One single electron bunch was used for charges below $50 \mathrm{pC}$; for higher charges multiple bunches with 154 ns temporal spacing were used.

The curves show a linear behavior up to a certain saturation threshold. A linear fit has been applied to all curves to evaluate the photons/sr/charge value (Table I). The error of the absolute calibration includes the errors from CCD counts, objective solid angle, fit accuracy, and charge measurement with the ICT. For comparison, the scintillation signal per solid angle calculated from the information in the work of Glinec et al. ${ }^{11}$ is also shown in Fig. 4. The performed calibration for the KODAK Lanex Fine screen of $(1.75 \pm 0.15)$ photons/sr/pC shows good agreement with the value obtained in Ref. 11 for 3-9 MeV electrons of $(1.54 \pm 0.19)$ photons $/ \mathrm{sr} / \mathrm{pC}$. This confirms that the scintillation signal is independent of electron energy above $3 \mathrm{MeV}$ and also that the directional characteristic can be well estimated by a Lambertian law.

\section{B. Cross-calibration against CLS}

To simplify recalibration after changes to the optical setup, the scintillation signal from the screen is referenced to

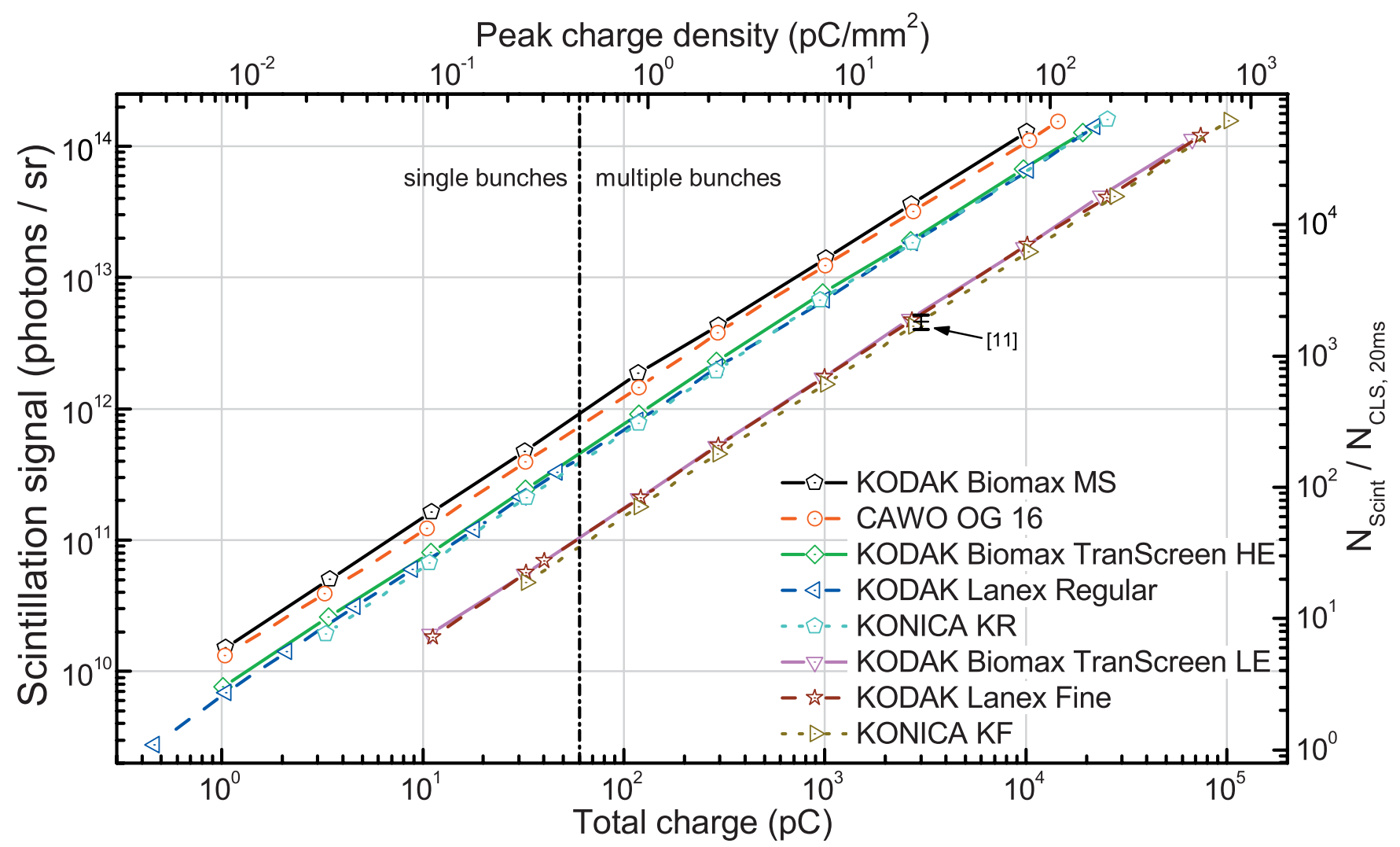

FIG. 4. (Color) Absolute calibration of the scintillation screens (left axis) and number of counts from the scintillation signal divided by the counts from the CLS (with 20 ms exposure time) as cross-calibration (right axis). The black cross shows the calculated value for KODAK Lanex Fine from Glinec et al. (Ref. $11)$. 
the CLS (see Sec. II B). The CCD counts from the scintillation screen $\mathrm{N}_{\text {Scint }}$ are divided by the counts from the CLS in $20 \mathrm{~ms}$ exposure time $\mathrm{N}_{\mathrm{CLS}, 20 \mathrm{~ms}}$. The ratio between the two signals is now independent from the optical setup. Thus, the calibration can be verified in an easy way regularly and after changes in the imaging system have been made.

The light emitted by the CLS is very weak; therefore the images for the CLS counts should be taken separately with a long exposure time $(\sim 1 \mathrm{~s})$ to get a good signal-to-noise ratio. Afterward, the CLS counts have to be rescaled to $20 \mathrm{~ms}$ exposure time to enable comparison to the counts from the scintillating screen. The ratio $\mathrm{N}_{\text {Scint }} / \mathrm{N}_{\mathrm{CLS}, 20 \mathrm{~ms}}$ is also plotted in Fig. 4, and the calibration parameters are given in Table I. For example, when taking images with $20 \mathrm{~ms}$ exposure time and the CAWO OG 16 screen, $1 \mathrm{pC}$ will result in 4.86 times the signal of the CLS, or equivalently the CLS signal corresponds to $0.206 \mathrm{pC}$ charge, respectively.

These calibration values can be easily adapted to other setups by transferring the CLS and placing it in the same image as the scintillating signal that is to be measured. Other CLSs can be calibrated by comparing the absolute photon number. The main error for this calibration stems from the charge measurement with the ICT, which is accurate to within 3\% of the signal. The counts of the CLS and the electron signal on the scintillating screen are accurate to about $1 \%$.

The absolute photon number emitted by the CLS at the time of the measurement can be estimated by this crosscalibration. The light emitted by the CLS has a spectrum extending from 450 to $650 \mathrm{~nm}$ (Fig. 3). The estimated quantum efficiency for this spectrum is $(21.6 \pm 1.5) \%$. The absolute photon flux from the CLS is determined with this information and the counts from the CCD camera image to be $(1.33 \pm 0.17) 10^{8}$ photons $/ \mathrm{sr} / \mathrm{ms}$.

\section{Saturation effects of scintillating screens}

For high charge densities on the scintillating screens, saturation effects were visible. Figure 5(a) shows saturation in the measured peak charge density with the screen CAWO OG 16. The charge density $\rho_{\mathrm{ICT}}$ applied to the screens is calculated with the charge measured by the ICT and the Gaussian fit of the electron peak [Fig. 1(c)]. $\rho_{\text {scint }}$ is the charge density measured on the scintillating screen. The saturation is fitted with Birk's law ${ }^{19}$ for saturation of scintillators,

$$
\rho_{\text {scint }}=\frac{\rho_{\text {real }}}{1+\mathrm{B} \rho_{\text {real }}},
$$

which describes the reduction of the measured scintillator signal with increasing charge density. Equation (1) is fitted to all the measured data of all screens to quantify possible saturation. Deviations from the linear fit were observed for the four screens with the most intense scintillation signal [Fig. $5(\mathrm{~b})]$.

A saturation threshold $\rho_{\text {sat }}$ is defined as the charge density, at which $\rho_{\text {scint }}$ will have dropped to $95 \%$ of the linear fit. The saturation thresholds for all screens are given in Table I. For the screens with less signal, the measurements could
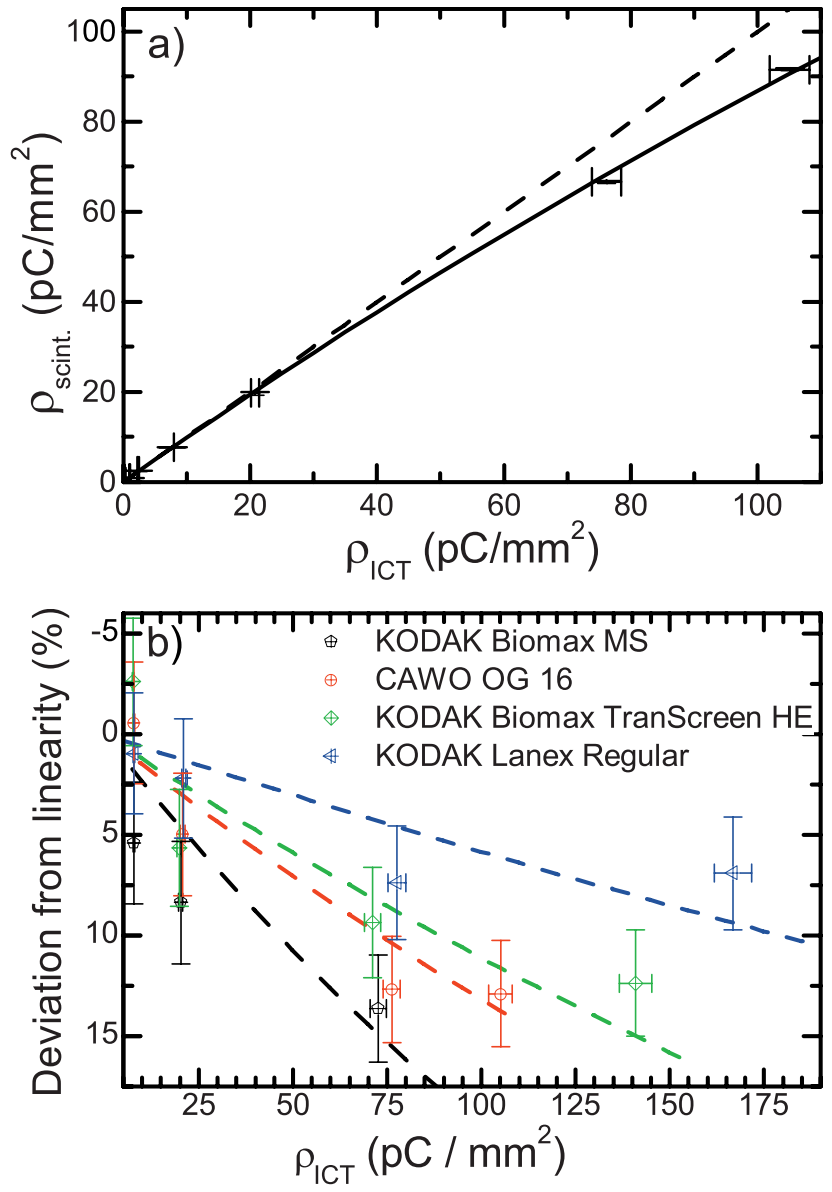

FIG. 5. (Color) (a) Example for measured charge density in the peak from the scintillation screen (CAWO OG 16) vs the charge density calculated via the charge measured by the ICT and the Gaussian fit, fitted linearly below saturation (dashed line) and with Birk's saturation law [Eq. (1)] (solid line). (b) Relative deviation of $\rho_{\text {scint }}$ from the linear fit to all data points for the four screens where saturation was observed. The dashed line shows the fit with the nonlinear part of Eq. (1). Only the points with the highest charge density are shown.

only be performed to the onset of saturation. It is therefore only possible to give a lower limit for the saturation threshold for these screens.

The calculation of saturation assumes that the whole charge arrives in temporal window that is much shorter than the decay time of the scintillation screen of $1 \mathrm{~ms} .{ }^{20}$ This is not valid any more for high charge densities and thus long pulse trains. For the screens KODAK Biomax TranScreen LE, KODAK Lanex Fine, and KONICA KF, the signal has already decayed to $10 \%-20 \%$ until the last electron bunch arrives. This will cause a reduction in the measured saturation effect; the real saturation thresholds $\rho_{\text {sat }}$ for these screens might therefore be smaller than observed in the measurements. However, the lower limit for $\rho_{\text {sat }}$ is estimated to be $100 \mathrm{pC} / \mathrm{mm}^{2}$ because below this charge density, the electron bunch duration was less than $5 \%$ of the scintillator decay time and thus short enough to detect possible saturation.

The light emission of the scintillating screens is temperature-dependent. However, even for the highest electron beam flux used in the calibration measurement, the screen can only heat up in the millikelvin range due to the energy deposited by one electron pulse and has enough time 
to cool down before the next pulse. The saturation can therefore not be attributed to thermal quenching. Changes in the room temperature, however, can influence the light emission by about $0.5 \% / \mathrm{K}^{21}$

\section{CONCLUSION}

We present a new absolute charge calibration measurement and linearity test for various scintillating screens for relativistic electron detection with a dynamic range and accuracy that has not been done so far to our knowledge. A linear response was confirmed over typically four orders of magnitude. The calibration measurement was performed at the ELBE linear accelerator delivering electron pulses with 2 ps duration, $40 \mathrm{MeV}$ energy, and variable charge. The absolute photon number per solid angle and charge were measured for all screens over four orders of magnitude (Table I). The signal per charge varies over one order of magnitude for the different screens. For the screen with the strongest signal (KODAK Biomax MS), the lower detection limit in this calibration measurement was about $0.5 \mathrm{pC}$ in a spot with $11 \mathrm{~mm}$ FWHM, corresponding to charge densities on the order of $10 \mathrm{fC} / \mathrm{mm}^{2}$. In LWFA experiments the laser light has to be shielded carefully to reach this detection threshold.

Saturation effects were observed starting at charge densities of around $20 \mathrm{pC} / \mathrm{mm}^{2}$ for some scintillators. Screens with a lower signal per unit charge also show a higher saturation threshold $\rho_{\text {sat. }}$. Kodak Biomax MS and CAWO OG 16 show the highest signal per unit charge and are therefore the optimum screens for applications with low charge. The onset of saturation around $20 \mathrm{pC} / \mathrm{mm}^{2}$ is still quite high for typical laser wakefield experiments, where charges of the order of 1-100 pC are dispersed in an ESM over several $\mathrm{cm}^{2}$. Additionally, even when the saturation threshold $\rho_{\text {sat }}$ is exceeded in the experiment, the measured charge density $\rho_{\text {scint }}$ can be afterward converted with Eq. (1) to the real charge density $\rho_{\text {real }}$ with good accuracy if $\rho_{\text {scint }}$ is not much higher than $\rho_{\text {sat }}$. With present laser accelerators, the saturation limits might only be exceeded by using magnetic lenses to focus the accelerated electrons ${ }^{22}$ or placing the scintillating screen close to the electron source.

The scintillation signal is referenced to the signal generated by a CLS emitting $(1.33 \pm 0.17) \times 10^{8} \mathrm{photons} / \mathrm{sr} / \mathrm{ms}$. Thus, the absolute calibration can be easily adapted to other electron detection setups. The accuracy of this calibration is mainly determined by the careful evaluation of the photon counts from the CLS.

\section{ACKNOWLEDGMENTS}

This work was supported by DFG-Project Transregio TR 18, the Association EURATOM-Max-Planck-Institut für Plasmaphysik, the Munich Centre for Advanced Photonics (MAP), and the LASERLAB-EUROPE/LAPTECH, EC FP7, Contract No. 228334.

${ }^{1}$ T. Tajima and J. M. Dawson, Phys. Rev. Lett. 43, 267 (1979).

${ }^{2}$ J. Faure, Y. Glinec, A. Pukhov, S. Kiselev, S. Gordienko, E. Lefebvre, J.-P. Rousseau, F. Burgy, and V. Malka, Nature (London) 431, 541 (2004).

${ }^{3}$ C. G. R. Geddes, Cs. Toth, J. van Tilborg, E. Esarey, C. B. Schroeder, D. Bruhwiler, C. Nieter, J. Cary, and W. P. Leemans, Nature (London) 431, 538 (2004).

${ }^{4}$ S. P. D. Mangles, C. D. Murphy, Z. Najmudin, A. G. R. Thomas, J. L. Collier, A. E. Dangor, E. J. Divall, P. S. Foster, J. G. Gallacher, C. J. Hooker, D. A. Jaroszynski, A. J. Langley, W. B. Mori, P. A. Norreys, F. S. Tsung, R. Viskup, B. R. Walton, and K. Krushelnick, Nature (London) 431, 535 (2004).

${ }^{5}$ W. P. Leemans, B. Nagler, A. J. Gonsalves, Cs. Toth, K. Nakamura, C. G. R. Geddes, E. Esarey, C. B. Schroeder, and S. M. Hooker, Nat. Phys. 2, 696 (2006).

${ }^{6}$ J. Faure, C. Rechatin, A. Norlin, A. Lifschitz, Y. Glinec, and V. Malka, Nature (London) 444, 737 (2006).

${ }^{7}$ S. Karsch, J. Osterhoff, A. Popp, T. P. Rowlands-Rees, Zs. Major, M. Fuchs, B. Marx, R. Hörlein, K. Schmid, L. Veisz, S. Becker, U. Schramm, B. Hidding, G. Pretzler, D. Habs, F. Grüner, F. Krausz, and S. M. Hooker, New J. Phys. 9, 415 (2007).

${ }^{8}$ K. Schmid, L. Veisz, F. Tavella, S. Benavides, R. Tautz, D. Herrmann, A. Buck, B. Hidding, A. Marcinkevicius, U. Schramm, M. Geissler, J. Meyer-ter-Vehn, D. Habs, and F. Krausz, Phys. Rev. Lett. 102, 124801 (2009).

${ }^{9}$ W. S. Graves and E. D. Johnson, "A high resolution electron beam profile monitor," Proceedings of the Particle Accelerator Conference 1997, Vancouver, British Columbia, Canada (1997), pp. 1993-1995.

${ }^{10}$ K. Nakamura, W. Wan, N. Ybarrolaza, D. Syversrud, J. Wallig, and W. P. Leemans, Rev. Sci. Instrum. 79, 053301 (2008).

${ }^{11}$ Y. Glinec, J. Faure, A. Guemnie-Tafo, V. Malka, H. Monard, J. P. Larbre, V. De Waele, J. L. Marignier, and M. Mostafavi, Rev. Sci. Instrum. 77, 103301 (2006)

${ }^{12}$ B. Hidding, G. Pretzler, M. Clever, F. Brandl, F. Zamponi, A. Lübcke, T. Kämpfer, I. Uschmann, E. Förster, U. Schramm, and R. Sauerbrey, Rev. Sci. Instrum. 78, 083301 (2007).

${ }^{13}$ S. Masuda, E. Miura, K. Koyama, and S. Kato, Rev. Sci. Instrum. 79, 083301 (2008).

${ }^{14}$ K. A. Tanaka, T. Yabuuchi, T. Sato, R. Kodama, Y. Kitagawa, and T. Takahashi, Rev. Sci. Instrum. 76, 013507 (2005).

${ }^{15}$ C. Gahn, G. D. Tsakiris, K. J. Witte, P. Thirolf, and D. Habs, Rev. Sci. Instrum. 71, 1642 (2000).

${ }^{16}$ K. Zeil, S. D. Kraft, A. Jochmann, F. Kroll, W. Jahr, U. Schramm, L. Karsch, J. Pawelke, B. Hidding, and G. Pretzler, Rev. Sci. Instrum. 81, 013307 (2010).

${ }^{17}$ G. E. Giakoumakis and D. M. Miliotis, Phys. Med. Biol. 30, 21 (1985).

${ }^{18}$ Information from mb-microtec.

${ }^{19}$ J. B. Birks, The Theory and Practice of Scintillation Counting (Pergamon, Oxford, 1964).

${ }^{20}$ Information from KODAK and CAWO.

${ }^{21}$ E. I. Gorokhova, V. A. Demidenko, O. A. Khristich, S. B. Mikhrin, and P. A. Rodnyi, J. Opt. Technol. 70, 10 (2003).

${ }^{22}$ T. Eichner, F. Grüner, S. Becker, M. Fuchs, D. Habs, R. Weingartner, U. Schramm, H. Backe, P. Kunz, and W. Lauth, Phys. Rev. ST Accel. Beams 10, 082401 (2007). 